\title{
Gemcitabine plus nab-paclitaxel for locally advanced or borderline resectable pancreatic cancer
}

\begin{abstract}
Akiko Tsujimoto ${ }^{1}$, Kentaro Sudo $^{1 *}$, Kazuyoshi Nakamuraa ${ }^{1}$, Emiri Kita ${ }^{1}$, Ryusuke Hara $^{2}$, Wataru Takayama ${ }^{3}$, Hiroshi Ishii ${ }^{1}$ \& Taketo Yamaguchi $^{1}$

Overall survival in a phase III study for metastatic pancreatic cancer has significantly improved with gemcitabine (GEM) plus nab-paclitaxel. However, to date, there is limited data on the efficacy and safety of its use for patients with locally advanced (LA) or borderline resectable pancreatic cancer (BRPC). Here, we investigated the efficacy and safety of first-line GEM plus nab-paclitaxel for LA or $B R P C$. We retrospectively analysed consecutive patients with pathologically confirmed, untreated LA or BRPC who started receiving first-line GEM plus nab-paclitaxel. A total of 30 patients ( $L A, n=22$; $B R P C, n=8)$ were analysed. Twelve patients $(40 \%)$ without distant metastasis received additional chemoradiotherapy using S-1. Laparotomy was performed on 8 patients and $6(20 \% ; L A, n=3 ; B R$, $\mathrm{n}=3$ ) achieved $\mathrm{R} 0$ resection. Objective response rate was $44.8 \%$. For all patients, median progressionfree survival and overall survival were 14.8 and 29.9 months, respectively. Median overall survival for LA was 24.1 months with a 2-year survival rate of $50.8 \%$. The most frequently observed grade 3 or 4 toxicities were neutropenia (73\%) and biliary infection (13\%). First-line GEM plus nab-paclitaxel was well-tolerated and feasible with an encouraging survival for LA or BRPC.
\end{abstract}

Locally advanced (LA) or borderline resectable pancreatic cancer (BRPC) are subsets of PC which account for about $30-40 \%$ of all patients ${ }^{1,2}$. Patterns of disease progression and survival outcomes differ from those with metastatic disease $\mathrm{e}^{1,3}$. To date, systemic chemotherapy, combined or not with chemoradiotherapy (CRT), represents the standard treatment for LAPC 4 . In addition, neoadjuvant therapy followed by surgical resection is the preferred option in BRPC, despite a lack of high-level evidence ${ }^{5}$.

However, it remains unclear what is the best first-line therapy in LA or BRPC patients. Up to the early 2010s, CRT or gemcitabine (GEM)-based systemic chemotherapy were the mainstay in the treatment of LAPC. However, it has been shown that those conventional therapies provided limited survival benefits, having a reported median survival of 8-17 months ${ }^{6-12}$. Consequently, it is urgent to develop more effective therapies.

In a large-scale, international phase III study for metastatic pancreatic cancer (MPACT), it has been shown that GEM plus nab-paclitaxel significantly improved both overall survival and progression-free survival (PFS) compared to GEM alone ${ }^{13}$. Additionally, an improved radiographic response was observed (23\% vs. $7 \%$, $\mathrm{P}<0.001)$. As stated in the National Comprehensive Cancer Network (NCCN) guidelines of pancreatic adenocarcinoma, GEM plus nab-paclitaxel is currently accepted as an option in the management of LA or BRPC 5 . However, to date, there is limited data available on the safety and efficacy of its use for this population. To address this issue, we retrospectively investigated the efficacy and safety of first-line GEM plus nab-paclitaxel for LA or BRPC in the present study.

\section{Results}

Patients. A total of 196 patients with pancreatic cancer started chemotherapy at the division of Gastroenterology of Chiba Cancer Centre between January 2015 and August 2017 (Fig. 1). Of the 196 patients, 47 patients had LA or BR disease at the initiation of chemotherapy. Of these, 17 patients were excluded due to receiving other treatment $(n=15)$, starting GEM plus nab-paclitaxel at the referring hospital $(n=1)$ or lack of pathologic confirmation $(n=1)$. Thus, 30 patients with LA unresectable $(n=22)$ and BRPC $(n=8)$ were eligible for the study (Fig. 1). Table 1 shows the baseline characteristics of the study population. The median age was 67

${ }^{1}$ Division of Gastroenterology, Chiba Cancer Centre, Chiba, Japan. ${ }^{2}$ Division of Radiation Oncology, Chiba Cancer Centre, Chiba, Japan. ${ }^{3}$ Division of Hepatobiliary and Pancreatic Surgery, Chiba Cancer Centre, Chiba, Japan. *email: kentarosudo9@yahoo.co.jp 


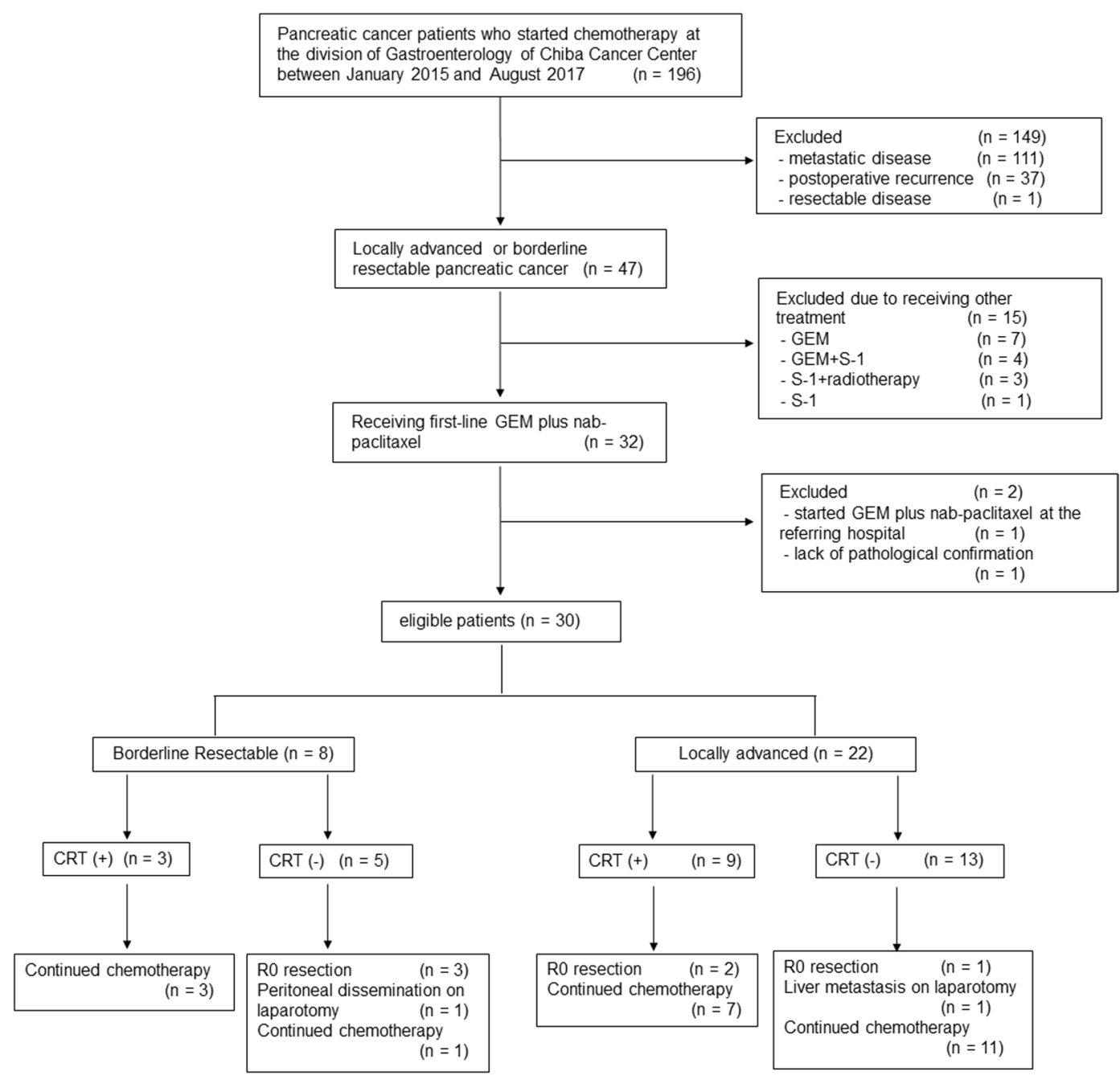

Figure 1. Consort Diagram.

years. All patients had a good performance status (PS) of 0 or 1 . The primary tumour was located in the head of the pancreas in 18 patients $(60 \%)$ and it was in the body/tail in $12(40 \%)$. Biliary drainage was required prior to treatment initiation in 16 patients (53\%). Specifically, 14 patients underwent endoscopic biliary stent placement and 2 underwent choledochojejunostomy. The median tumour size was $44 \mathrm{~mm}$. Median serum CA19-9 concentration was $160 \mathrm{U} / \mathrm{mL}$.

Treatment. Figure 1 summarizes the treatment for all the patients. The initial doses of GEM or nab-paclitaxel were reduced by more than $10 \%$ in 4 patients $(13.3 \%)$ because of repeated biliary tract infection $(n=1)$, patient's wish $(n=1)$, co-existing disease $(n=1)$ or physician's discretion $(n=1)$. The remaining patients $(n=26)$ started GEM plus nab-paclitaxel at standard dosage. Treatment was terminated due to adverse events (AEs) in 2 patients. The median duration for first-line GEM plus nab-paclitaxel was 5.4 months (range: 1.4-25.8 months). In total, $90 \%(n=27)$ of the patients required dose modification (dose reduction, $n=12$; treatment schedule modification, $\mathrm{n}=3$; both, $\mathrm{n}=12$ ) owing to the development of toxicity. Forty-three percent of the patients had dose reduction of GEM and $80 \%$ had dose reduction of nab-paclitaxel. Of the 30 patients, 12 (40\%) received S-1-based CRT after the first-line GEM plus nab-paclitaxel, followed by maintenance chemotherapy using GEM plus nab-paclitaxel $(\mathrm{n}=4)$, S-1 alone $(\mathrm{n}=5)$ or GEM plus $\mathrm{S}-1(\mathrm{n}=1)$. The median duration from the start of GEM plus nab-paclitaxel to CRT initiation was 6 months (range, 2.8-8.5 months). The median duration from the completion of CRT to the start of maintenance chemotherapy was 2.5 weeks (range, $1.1-4$ weeks).

Safety. All the patients $(n=30)$ were evaluable for AEs. Table 2 shows Grade 3 or 4 AEs observed during the first-line GEM plus nab-paclitaxel. The most common Grade 3 or 4 AE were neutropenia ( $73 \%$ of the patients), followed by biliary infection ( $13 \%$ of patients). All the patients who developed biliary infection had pancreatic head tumour and had undergone biliary stent placement. Among these patients, one patient developed acute cholecystitis during chemotherapy with GEM plus nab-paclitaxel. Repeated biliary tract infection after biliary stent placement was the main cause of acute cholecystitis. The patient required percutaneous gallbladder drainage 


\begin{tabular}{|c|c|}
\hline & n or median (range) \\
\hline $\mathrm{n}$ & 30 \\
\hline \multicolumn{2}{|l|}{ Gender } \\
\hline Male & 14 \\
\hline Female & 16 \\
\hline Median Age, years (range) & $67(47-75)$ \\
\hline \multicolumn{2}{|l|}{ ECOG PS } \\
\hline 0 & 16 \\
\hline 1 & 14 \\
\hline \multicolumn{2}{|l|}{ Site of primary tumour } \\
\hline Head & 18 \\
\hline Body or tail & 12 \\
\hline \multicolumn{2}{|l|}{ Biliary drainage } \\
\hline Stent & 14 \\
\hline Bypass & 2 \\
\hline Median tumour size, mm (range) & $44(25-88)$ \\
\hline \multicolumn{2}{|l|}{ Resectability } \\
\hline $\begin{array}{l}\text { BR } \\
\text { LA }\end{array}$ & $\begin{array}{l}8 \\
22\end{array}$ \\
\hline \multicolumn{2}{|l|}{ Vessel invasion, (\%) } \\
\hline SMA & $24(80 \%)$ \\
\hline $\mathrm{CA}$ & $15(50 \%)$ \\
\hline $\mathrm{HA}$ & $18(60 \%)$ \\
\hline $\mathrm{SMV} / \mathrm{PV}$ & $25(83.3 \%)$ \\
\hline CA19-9 (U/mL), median [range] & $160[<2-44074]$ \\
\hline
\end{tabular}

Table 1. Patient characteristics. Abbreviations: ECOG PS, Eastern Cooperative Oncology Group performance status; BR, borderline resectable; LA, locally advanced; SMA, superior mesenteric artery; CA, celiac axis; HA, hepatic artery; SMV, superior mesenteric vein; PV, portal vein.

\begin{tabular}{|l|l|}
\hline & n (\%) \\
\hline White blood cell decreased & $5(17 \%)$ \\
\hline Neutrophil count decreased & $22(73 \%)$ \\
\hline Febrile neutropenia & 0 \\
\hline Anaemia & $2(7 \%)$ \\
\hline Anorexia & $1(3 \%)$ \\
\hline Nausea & $1(3 \%)$ \\
\hline Biliary tract infection & $4(13 \%)$ \\
\hline Pneumonitis & $1(3 \%)$ \\
\hline Lung infection & $1(3 \%)$ \\
\hline Haematuria & $1(3 \%)$ \\
\hline Fatigue & 0 \\
\hline Peripheral neuropathy & 0 \\
\hline Diarrhoea & 0 \\
\hline
\end{tabular}

Table 2. Grade 3 or 4 adverse events.

followed by elective cholecystectomy. Biliary tract infection in other patients was mainly induced by stent occlusion, and this was managed by stent replacement and antibiotic treatment.

Furthermore, two patients had additional AEs. One patient developed interstitial lung disease, likely induced by GEM, the other developed pneumocystis pneumonia. Both patients recovered with conservative therapy. We did not observe cases of febrile neutropenia or treatment-related death.

Response. Twenty-nine patients were evaluable for radiographic response. One patient was excluded from the evaluation of objective response due to the lack of a measurable lesion. During chemotherapy with GEM plus nab-paclitaxel, 13 patients (44.8\%) achieved a partial response, $15(51.7 \%)$ had stable disease and $1(3.4 \%)$ developed disease progression. Among the 15 patients with stable disease, 2 showed a partial response after additional CRT. Therefore, 15 patients (51.7\%) achieved a partial response following multimodal treatment using GEM plus nab-paclitaxel. 
Overall, 8 patients $(26.7 \%$; BR, $\mathrm{n}=4 ; \mathrm{LA}, \mathrm{n}=4)$ underwent laparotomy with a curative intent (Fig. 1$)$. Three patients with BRPC received GEM plus nab-paclitaxel as neoadjuvant therapy, and they underwent either pancreatoduodenectomy $(n=2)$ or distal pancreatectomy $(n=1)$ at 2.2-5.6 months after treatment initiation. They all achieved R0 resection. Further, one patient with BRPC who showed partial response after receiving first-line GEM plus nab-paclitaxel therapy underwent laparotomy at 24.4 months after treatment initiation. However, this patient was found to be inoperable owing to peritoneal dissemination. Three patients with LAPC who showed significant radiographic response underwent either pancreatoduodenectomy $(n=2)$ or distal pancreatectomy $(\mathrm{n}=1)$ at 6.1-10.2 months after treatment initiation. They all achieved R0 resection. Of note, one patient treated with additional CRT showed a pathologic complete response. Another patient with LAPC underwent laparotomy at 3.4 months after treatment initiation, but this patient was found to be inoperable owing to liver metastasis.

Survival. The median follow-up length for censored cases was 25.2 months (range: 6.9-35.3 months). Disease progression was observed in 19 patients. The median PFS for all treated patients $(\mathrm{n}=30)$ was 14.8 months $(95 \%$ CI, 11.4-24.4) (Fig. 2A). Specifically, median PFS for LA $(\mathrm{n}=22)$ and BRPC patients $(\mathrm{n}=8)$ was 12.4 months (95\% CI, 8.9-20.3) and 24.4 months (95\% CI, 11.4-NA), respectively (Fig. 2B).

For all treated patients $(n=30)$, the median overall survival was 29.9 months $(95 \%$ CI, 20.1-NA) with a 2-year survival rate of $65.1 \%(95 \% \mathrm{CI}, 43.4-80.2 \%)$ (Fig. 2C). For LA patients $(\mathrm{n}=22)$, median overall survival and survival rate at 2 years were 24.1 months (95\% CI, 17.9-NA) and 50.8\% (95\% CI, 26.1-71\%), respectively (Fig. 2D). The median overall survival of BR patients $(n=8)$ was not reached (Fig. 2D). The median overall survival for 24 patients who did not undergo pancreatectomy was 24.9 months (95\% CI, 18.6-NA) with a 2-year survival rate of 58\% (95\% CI, 34.4-75.7\%) (Fig. 2E).

\section{Discussion}

There is limited data available as to the efficacy and safety of GEM plus nab-paclitaxel in patients with LA or BRPC. Gulhati et al. assessed the use of first-line GEM plus nab-paclitaxel for the treatment of localised pancreatic cancer in a large case series $(n=99)^{14}$. In that study, most of the patients $(81 \%)$ received biweekly doses of GEM plus nab-paclitaxel. The median survival of all the patients, including those with resectable pancreatic cancer $(n=45)$, was 18 months, but specific survival data for patients with BR $(n=14)$ or LAPC $(n=40)$ have not been presented.

In the present study, we demonstrated that first-line GEM plus nab-paclitaxel was feasible. Additionally, we showed that this line of treatment is associated with an encouraging survival outcome for this population. Median overall survival of patients treated with GEM plus nab-paclitaxel (all patients, 29.9 months; LA patients, 24.1 months) appeared to be better than for patients treated with conventional therapies (e.g. upfront CRT or GEM-based systemic chemotherapy) (8-17 months) (Table 3$)^{6-12}$. Furthermore, median overall survival of 24 patients $(80 \%)$ treated with non-surgical treatment alone reached 24.9 months.

It is possible that a better patient selection positively influenced the survival outcomes in the present study. However, previously published studies have shown similar survival outcomes by assessing FOLFIRINOX or GEM plus nab-paclitaxel regimens for LA or BRPC (Table 4) $)^{14-17}$. FOLFIRINOX has also shown a significant improvement of overall survival compared to GEM alone in metastatic pancreatic cancer ${ }^{18}$. Importantly, both in our study and in previously published data, a multimodal approach centring on potent systemic therapies was employed. Following the use of systemic chemotherapy, $40-80 \%$ of patients received CRT and $20-40 \%$ underwent surgical resection (Table 4). Such data indicate that multimodal treatment using these novel regimens provide significant survival benefits (median overall survival, 20-30 months). Limited evidence supports this strategy and the clinical significance of CRT is still controversial. However, we believe that the improved survival outcomes warrant further investigation.

Objective response rate of GEM plus nab-paclitaxel (44.8\%) was higher than conventional regimens for LAPC (5-41\%) (Table 3). These results are consistent with those of the phase I/II study of GEM plus nab-paclitaxel for metastatic pancreatic cancer reported by Ueno et al. The authors showed that $69 \%$ of the patients (18 out of 26) underwent $>30 \%$ shrinkage of pancreatic tumour ${ }^{19}$. In the era of neoadjuvant or conversion strategy for localised pancreatic cancer, first-line chemotherapy with higher response rate represents an attractive option.

In the present study, we found that first-line GEM plus nab-paclitaxel was well-tolerated and feasible in patients with LA or BRPC. The most common Grade 3 or 4 toxicity was neutropenia. However, patients rarely developed life-threatening infections or febrile neutropenia. The frequency of biliary tract infection (13\%) was comparable to those observed in our previous prospective study $(20 \%)^{20}$. The most common non-haematological toxicity was peripheral neuropathy in prospective studies ${ }^{13,19}$. In contrast, none of the patients developed grade 3 or more peripheral neuropathy in this study, possibly because the dosage of nab-paclitaxel was reduced or its administration was temporally discontinued when grade 2 peripheral neuropathy was observed.

The present study had some limitations. The main one is its retrospective nature. Thus, no standardized indication of pancreatectomy after induction of GEM plus nab-paclitaxel in BRPC was available. Furthermore, additional CRT was not performed according to predefined criteria. Radiographic evaluation was not performed according to the protocol specified duration. Consequently, objective response rate and PFS were biased. Finally, we enrolled a small number of patients, and the sample size was not calculated based on any statistical hypotheses.

The present study has an important fundamental strength. It included patients who started receiving first-line GEM plus nab-paclitaxel for LA or BRPC at our institution in a consecutive manner. We analysed all the treated patients, whether or not they received CRT or pancreatectomy based on an intention-to-treat basis. Furthermore, to avoid selection bias, we excluded from the study those patients who were referred from other institutions after the introduction of GEM plus nab-paclitaxel. Thus, the present study can provide precise data on survival outcomes of first-line GEM plus nab-paclitaxel for LA or BRPC in clinical practice. 
A

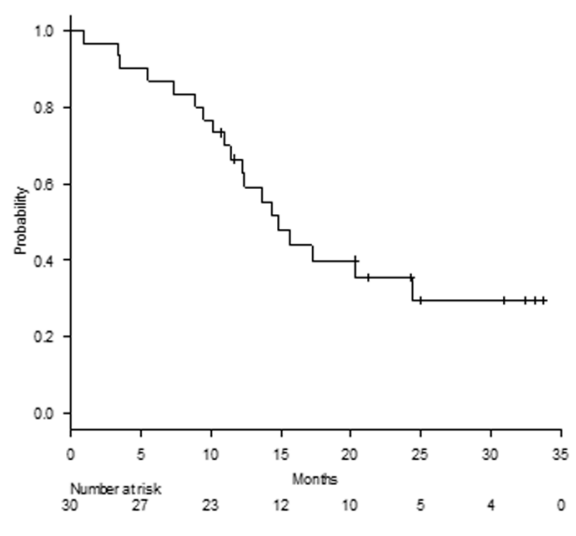

C

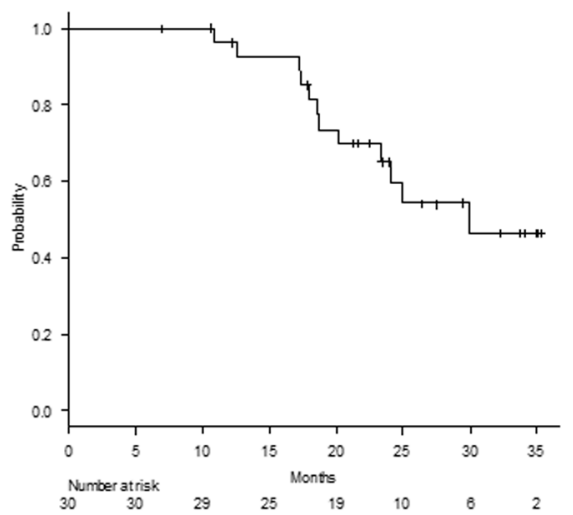

E

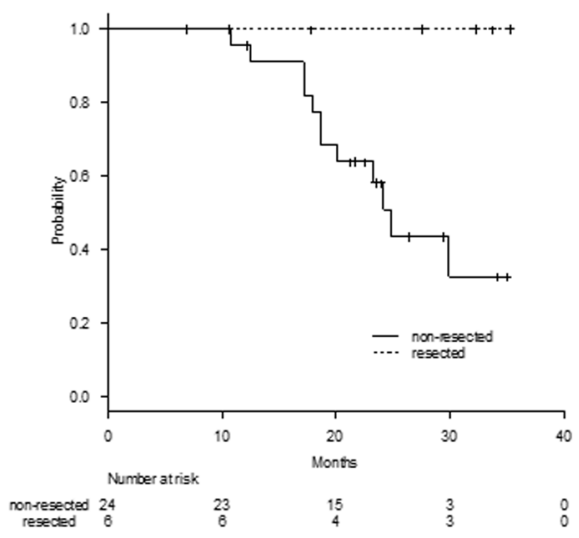

B
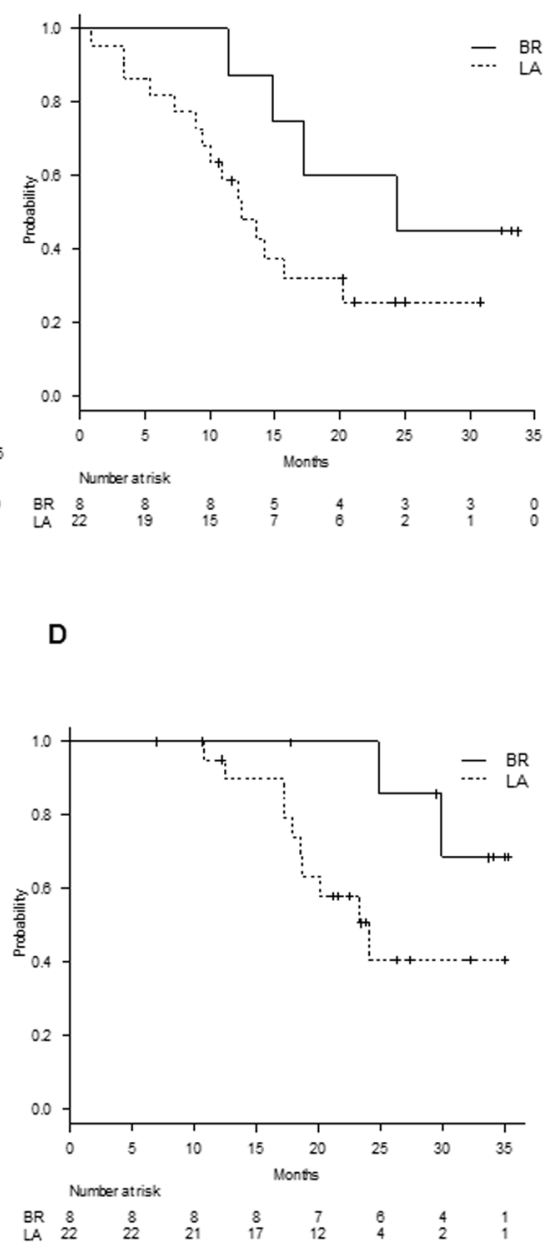

Figure 2. (A) Progression-free survival for all patients $(n=30)$ and $(B)$ for $L A(n=22)$ and $B R(n=8)$ patients. (C) Overall survival curves of all patients $(n=30)$ and (D) LA $(n=22)$ and BR $(n=8)$ patients. (E) Overall survival for resected $(n=6)$ and non-resected $(n=24)$ patients.

In conclusion, our results demonstrate that first-line GEM plus nab-paclitaxel was well-tolerated and feasible in patients with LA or BRPC. Using a multimodal approach, we observed good survival outcomes (median survival of 29.9 months in all patients and 24.1 months in patients with LA). Although the study is limited by its retrospective nature with a small sample size, these results were better than those of patients treated with conventional therapies (e.g. upfront CRT or GEM-based chemotherapy). Further prospective studies are warranted to elucidate the effectiveness of first-line GEM plus nab-paclitaxel in a large cohort of patients. 


\begin{tabular}{|c|c|c|c|c|c|c|c|}
\hline Author & design & CRT or systemic chemotherapy & $\mathbf{n}$ & $\begin{array}{l}\text { Response } \\
\text { rate }\end{array}$ & $\begin{array}{l}\text { PFS or TTP } \\
\text { (months) }\end{array}$ & $\begin{array}{l}\text { MST } \\
\text { (months) }\end{array}$ & ref. \\
\hline Ishii & P II & 5-FU + RT (50.4 Gy) & 20 & $10 \%$ & 4.9 & 10.3 & 6 \\
\hline Sudo & P II & S-1 + RT (50.4Gy) & 34 & $41 \%$ & 8.7 & 16.8 & 7 \\
\hline Okusaka & P II & GEM + RT (50.4Gy) & 42 & $21 \%$ & 4.4 & 9.5 & 8 \\
\hline Loehrer & P III & $\begin{array}{l}\text { GEM + RT (50.4Gy) } \\
\text { GEM }\end{array}$ & $\begin{array}{l}34 \\
37 \\
\end{array}$ & $\begin{array}{l}6 \% \\
5 \% \\
\end{array}$ & $\begin{array}{l}6.0 \\
6.7 \\
\end{array}$ & $\begin{array}{l}11.1 \\
9.2 \\
\end{array}$ & 9 \\
\hline Chauffert & P III & $\begin{array}{l}\text { 5-FU + Cisplatin + RT (60 Gy) } \\
\text { GEM }\end{array}$ & $\begin{array}{l}59 \\
60 \\
\end{array}$ & $\begin{array}{l}\text { NA } \\
\text { NA }\end{array}$ & $\begin{array}{l}14 \%^{\mathrm{a}} \\
32 \%^{\mathrm{a}} \\
\end{array}$ & $\begin{array}{ll}8.6 \\
13.0\end{array}$ & 10 \\
\hline Ishii & P II & GEM & 50 & NA & 6.0 & 15.0 & 11 \\
\hline Ueno & P III & $\begin{array}{l}\text { GEM + S-1 } \\
\text { GEM } \\
\text { S-1 }\end{array}$ & $\begin{array}{l}68 \\
66 \\
68\end{array}$ & $\begin{array}{l}\text { NA } \\
\text { NA } \\
\text { NA }\end{array}$ & $\begin{array}{l}\text { NA } \\
\text { NA } \\
\text { NA }\end{array}$ & $\begin{array}{l}15.9 \\
12.7 \\
13.8\end{array}$ & 12 \\
\hline
\end{tabular}

Table 3. Clinical studies of conventional CRT or GEM based chemotherapy for LAPC. Abbreviations: P II, phase II study; P III, phase III study; GEM, gemcitabine; CRT, chemoradiotherapy; RT, radiation therapy; PFS, progression-free survival; TTP, time to progression; MST, median survival time; NA, not available. ${ }^{\mathrm{a} O n e-y e a r}$ progression-free survival.

\begin{tabular}{|l|l|l|l|l|l|l|l|l|l|}
\hline Author & Design & Treatment & n & BR/LA & CRT & $\begin{array}{l}\text { Ro/1 } \\
\text { resection }\end{array}$ & $\begin{array}{l}\text { PFS } \\
\text { (months) }\end{array}$ & $\begin{array}{l}\text { MST } \\
\text { (months) }\end{array}$ & ref. \\
\hline Suker & Meta-analysis & FOLFIRINOX & 315 & $0 / 315$ & $64 \%^{\mathrm{a}}$ & $26 \%^{\mathrm{b}}$ & 15.0 & 24.2 & 15 \\
\hline Stein & Phase II & FOLFIRINOX & 31 & $11 / 20$ & $55 \%$ & $42 \%$ & 17.8 & 26.6 & 16 \\
\hline Gulhati & retrospective & GEM + nab-paclitaxel & 99 & $45^{\mathrm{c}} / 14 / 40$ & $45 \%$ & $15 \%$ & $11.0^{\mathrm{d}}$ & 18 & 14 \\
\hline Reni & Randomised phase II & $\begin{array}{l}\text { PAXG } \\
\text { GEM + nab-paclitaxel }\end{array}$ & $\begin{array}{l}26 \\
28\end{array}$ & $\begin{array}{l}10 / 16 \\
15 / 13\end{array}$ & $\begin{array}{l}88 \% \\
57 \%\end{array}$ & $\begin{array}{l}31 \% \\
32 \%\end{array}$ & $\begin{array}{l}12.5 \\
9.9\end{array}$ & $\begin{array}{l}20.7 \\
19.1\end{array}$ & 17 \\
\hline Current study & retrospective & GEM + nab-paclitaxel & 30 & $8 / 22$ & $40 \%$ & $20 \%$ & 14.8 & 29.9 & \\
\hline
\end{tabular}

Table 4. Published studies assessing GEM plus nab-paclitaxel or FOLFIRINOX for LA or BRPC. Abbreviations: FOLFIRINOX, fluorouracil, leucovorin, irinotecan and oxaliplatin; GEM, gemcitabine; PAXG, GEM, nabpaclitaxel, capecitabine and cisplatin; BR, borderline resectable; LA, locally advanced; CRT, chemoradiotherapy;

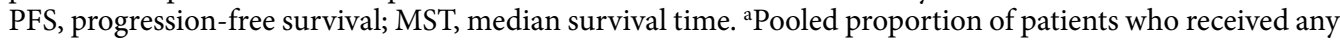
radiation therapy in a random-effects model. 'Pooled proportion of patients who had resection in a randomeffects model. cPotentially resectable. ${ }^{\mathrm{d}}$ Metastatic disease-free survival.

\section{Materials and Methods}

Patients. We reviewed medical records of consecutive patients with PC who started chemotherapy at the division of Gastroenterology of Chiba Cancer Centre between January 2015 and August 2017. The following are the selection criteria of the study population: (1) Patients who started receiving first-line GEM plus nab-paclitaxel in our institution; (2) Pathologically confirmed pancreatic adenocarcinoma; (3) No prior chemotherapy or radiotherapy for PC; and (4) LA unresectable or BRPC, based on the NCCN clinical practice guidelines in oncology version $2.2018^{21}$.

Patients who were referred from other institutions after the introduction of GEM plus nab-paclitaxel were excluded to avoid selection bias.

Treatment. Patients received intravenous nab-paclitaxel $125 \mathrm{mg} / \mathrm{m}^{2}$ followed by intravenous GEM $1000 \mathrm{mg} /$ $\mathrm{m}^{2}$ on days 1,8 , and 15 every 4 weeks. According to the patient's condition, GEM or nab-paclitaxel's doses were modified at the physician's discretion. Treatment was continued until the patient showed disease progression or unacceptable adverse events.

For patients who did not develop distant metastasis, we performed CRT using S-1 on the physician's discretion following a minimum of a 2-week washout period of GEM plus nab-paclitaxel. Further contraindications for additional CRT included tumour invasion to the gastrointestinal tract, huge tumours, massive lymph adenopathy or ascites. We performed S-1-based CRT as reported previously ${ }^{7,20}$. In brief, a total dose of 50.4 Gy was delivered in 28 fractions. S-1 was administered twice a day on days 1 to 14 and 22 to 35 . The daily S- 1 dose was determined according to body surface area (BSA) as follows: BSA $<1.25 \mathrm{~m}^{2}, 80 \mathrm{mg} / \mathrm{day} ; 1.25 \mathrm{~m}^{2} \leq \mathrm{BSA}<1.50 \mathrm{~m}^{2}, 100 \mathrm{mg} /$ day; and $1.50 \mathrm{~m}^{2} \leq \mathrm{BSA}, 120 \mathrm{mg} /$ day. Maintenance chemotherapy was started after CRT until the patient showed disease progression or unacceptable adverse events, as reported previously $\mathrm{y}^{7,20}$.

Indication for surgery was determined at the hepatobiliary pancreatic cancer board of Chiba Cancer Centre. Based on the radiographic findings and patient's conditions, indication for surgery required consensus among radiologists, surgeons, pathologists and medical oncologists.

Assessment. We evaluated the PS according to the ECOG (Eastern Cooperative Oncology Group) criteria. We assessed AEs according to the National Cancer Institute Common Terminology Criteria for Adverse Events, version 4. In the present study, we investigated AEs during first-line GEM plus nab-paclitaxel. We did not include AEs observed during maintenance chemotherapy after CRT. Radiographic responses were evaluated according to Response Evaluation Criteria in Solid Tumours, version 1.1. 
Statistical analysis. PFS was defined as the time between the date of treatment initiation and the date of disease progression or death. Overall survival was defined as the time between the date of treatment initiation and the date of death due to any cause. We used the Kaplan-Meier method to estimate the PFS and overall survival. The EZR ver. 1.35 software (https://cran.r-project.org/web/packages/RcmdrPlugin.EZR/index.html) was used to perform statistical analyses ${ }^{22}$.

Ethics statement. The ethical review board of Chiba Cancer Centre approved the study. We performed the study in compliance with the 1964 Helsinki declaration and its later amendments and with the ethical guidelines for medical research by the Ministry of Health, Labour and Welfare of Japan. For this type of study (retrospective, non-invasive, observational study), written informed consent is not required. We used our institutional official website as an opt-out method.

\section{Data availability}

The datasets generated and/or analysed during the current study are available from the corresponding author on reasonable request.

Received: 14 March 2019; Accepted: 18 October 2019;

Published online: 07 November 2019

\section{References}

1. Coveler, A. L., Herman, J. M., Simeone, D. M. \& Chiorean, E. G. Localized pancreatic cancer: multidisciplinary management. Am Soc Clin Oncol Educ Book 35, e217-e226 (2016).

2. Shaib, W. L. et al. Contemporary management of borderline resectable and locally advanced unresectable pancreatic cancer. Oncologist 21, 178-187 (2016).

3. Sultana, A. et al. Systematic review, including meta-analyses, on the management of locally advanced pancreatic cancer using radiation/combined modality therapy. Br J Cancer 96, 1183-1190 (2007).

4. Balaban, E. P. et al. Locally advanced, unresectable pancreatic cancer: American Society of Clinical Oncology Clinical Practice Guideline. J Clin Oncol 34, 2654-2668 (2016).

5. Tempero, M. A. et al. Pancreatic adenocarcinoma, version 2.2017, NCCN Clinical Practice Guidelines in Oncology. J Natl Compr Canc Netw 15, 1028-1061 (2017).

6. Ishii, H. et al. Protracted 5-fluorouracil infusion with concurrent radiotherapy as a treatment for locally advanced pancreatic carcinoma. Cancer 79, 1516-1520 (1997).

7. Sudo, K. et al. Phase II study of oral S-1 and concurrent radiotherapy in patients with unresectable locally advanced pancreatic cancer. Int J Radiat Oncol Biol Phys 80, 119-125 (2011).

8. Okusaka, T. et al. Phase II study of radiotherapy combined with gemcitabine for locally advanced pancreatic cancer. Br J Cancer $\mathbf{9 1}$, 673-677 (2004).

9. Loehrer, P. J. Sr et al. Gemcitabine alone versus gemcitabine plus radiotherapy in patients with locally advanced pancreatic cancer: an Eastern Cooperative Oncology Group trial. J Clin Oncol 29, 4105-4112 (2011).

10. Chauffert, B. et al. Phase III trial comparing intensive induction chemoradiotherapy (60 Gy, infusional 5-FU and intermittent cisplatin) followed by maintenance gemcitabine with gemcitabine alone for locally advanced unresectable pancreatic cancer. Definitive results of the 2000-01 FFCD/SFRO study. Ann Oncol 19, 1592-1599 (2008).

11. Ishii, H. et al. Phase II study of gemcitabine chemotherapy alone for locally advanced pancreatic carcinoma: JCOG0506. Jpn J Clin Oncol 40, 573-579 (2010).

12. Ueno, H. et al. Randomized phase III study of gemcitabine plus S-1, S- 1 alone, or gemcitabine alone in patients with locally advanced and metastatic pancreatic cancer in Japan and Taiwan: GEST study. J Clin Oncol 31, 1640-1648 (2013).

13. Von Hoff, D. D. et al. Increased survival in pancreatic cancer with nab-paclitaxel plus gemcitabine. N Engl J Med 369, 1691-1703 (2013).

14. Gulhati, P. et al. First-line gemcitabine and nab-paclitaxel chemotherapy for localized pancreatic ductal adenocarcinoma. Ann Surg Oncol 26, 619-627 (2019).

15. Suker, M. et al. FOLFIRINOX for locally advanced pancreatic cancer: a systematic review and patient-level meta-analysis. Lancet Oncol 17, 801-810 (2016).

16. Stein, S. M. et al. Final analysis of a phase II study of modified FOLFIRINOX in locally advanced and metastatic pancreatic cancer. Br J Cancer 114, 737-743 (2016).

17. Reni, M. et al. A randomised phase 2 trial of nab-paclitaxel plus gemcitabine with or without capecitabine and cisplatin in locally advanced or borderline resectable pancreatic adenocarcinoma. Eur J Cancer 102, 95-102 (2018).

18. Conroy, T. et al. FOLFIRINOX versus gemcitabine for metastatic pancreatic cancer. N Engl J Med 364, 1817-1825 (2011).

19. Ueno, H. et al. Phase I/II study of nab-paclitaxel plus gemcitabine for chemotherapy-naive Japanese patients with metastatic pancreatic cancer. Cancer Chemother Pharmacol 77, 595-603 (2016).

20. Sudo, K. et al. Phase II study of induction gemcitabine and S-1 followed by chemoradiotherapy and systemic chemotherapy using S-1 for locally advanced pancreatic cancer. Cancer Chemother Pharmacol 80, 195-202 (2017).

21. NCCN Clinical Practice Guidelines in Oncology version 2.2018 July 10, 2018. Available at, https:/www.nccn.org/professionals/ physician_gls/default.aspx.

22. Kanda, Y. Investigation of the freely available easy-to-use software 'EZR' for medical statistics. Bone Marrow Transplant 48, 452-458 (2013).

\section{Acknowledgements}

We would like to thank all patients involved in this study. We would like to thank Enago (www.enago.jp) for the English language review.

\section{Author contributions}

The study was designed by K.S. and A.T. Data collection was performed by K.S., A.T., K.N., E.K., R.H. and W.T. Statistical analysis was performed by K.S. and A.T. Data analysis and interpretation was performed by K.S., A.T., K.N., E.K., H.I. and T.Y. K.S. and T.A. wrote the manuscript. H.I. and T.Y. revised the manuscript. All authors reviewed the manuscript. 


\section{Competing interests}

Hiroshi Ishii reports honoraria from Yakult Honsha, Taiho Pharmaceutical, Eisai, TOWA and Teijin Pharma, and a consulting role for Ono Pharmaceutical, outside the submitted work. Other authors declare no conflict of interest.

\section{Additional information}

Correspondence and requests for materials should be addressed to K.S.

Reprints and permissions information is available at www.nature.com/reprints.

Publisher's note Springer Nature remains neutral with regard to jurisdictional claims in published maps and institutional affiliations.

(c) (i) Open Access This article is licensed under a Creative Commons Attribution 4.0 International License, which permits use, sharing, adaptation, distribution and reproduction in any medium or format, as long as you give appropriate credit to the original author(s) and the source, provide a link to the Creative Commons license, and indicate if changes were made. The images or other third party material in this article are included in the article's Creative Commons license, unless indicated otherwise in a credit line to the material. If material is not included in the article's Creative Commons license and your intended use is not permitted by statutory regulation or exceeds the permitted use, you will need to obtain permission directly from the copyright holder. To view a copy of this license, visit http://creativecommons.org/licenses/by/4.0/.

(C) The Author(s) 2019 